\title{
Patrones de prescripción de medicamentos antitiroideos en una población de Colombia
}

\section{Patterns of prescription of antithyroid drugs in a colombian population}

\author{
Jorge Enrique Machado-Alba, Diego Alejandro Medina-Morales, \\ Luis Fernando Valladales-Restrepo, Daniel Loaiza-Bedoya, \\ Milton Paredes-Mendoza • Pereira (Colombia)
}

\section{Resumen}

Introducción: en Colombia no se conoce la prevalencia de los trastornos asociados a tirotoxicosis ni se dispone de estudios fármacoepidemiológicos acerca de la prescripción de los medicamentos antitiroideos.

Objetivo: determinar los patrones de prescripción de los antitiroideos y variables asociadas a su uso en una población de pacientes en Colombia.

Métodos: estudio de corte transversal, realizado entre enero 1 y marzo 30 de 2015 sobre los hábitos de prescripción de medicamentos antitiroideos en una población afiliada al sistema de salud colombiano. Se midieron variables sociodemográficas, farmacológicas y de comedicación. Se diseñó una base de datos sobre el consumo de medicamentos y se utilizaron pruebas t de student, $X^{2}$ y modelos de regresión logística.

Resultados: un total de 327 pacientes en tratamiento con medicamentos antitiroideos fueron incluidos. La edad media fue de $53.7 \pm 18.1$ años y $78.3 \%$ de pacientes correspondió a mujeres. El metimazol se prescribió en $95.4 \%$ de los pacientes, el propiltiouracilo en $4.6 \%$. En $76.8 \%$ de pacientes se presentó comedicación; en particular con antihipertensivos $(38.2 \%)$ y adicionalmente con propranolol (34.3\%).

Conclusiones: la tendencia de prescripción de medicamentos antitiroideos en Colombia es similar a lo reportado en diferentes estudios a nivel mundial. El principal medicamento antitiroideo es metimazol, con una tasa de uso mayor a la reportada en Norteamérica y en estudios europeos. Las dosis del metimazol y de propiltiouracilo reportadas en este estudio se ajustan a las recomendaciones de la Asociación Americana de Endocrinología Clínica. (Acta Med Colomb 2018; 43: 150-155).

Palabras clave: hipertiroidismo; tirotoxicosis; antitiroideos; metimazol; propiltiouracilo; farmacoepidemiología, (DeCS).

\section{Abstract}

Introduction: the prevalence of disorders associated with thyrotoxicosis is not known in Colombia, nor pharmacoepidemiological studies are available on the prescription of antithyroid drugs.

Objective: to determine the prescription patterns of antithyroid drugs and variables associated with their use in a population of Colombian patients.

Methods: cross-sectional study, conducted between January 1 and March 30, 2015 on the prescription habits of antithyroid drugs in a population affiliated with the Colombian Health System. Sociodemographic, pharmacological and comedication variables were measured. A database on drug consumption was designed and student t-tests, $X^{2}$ and logistic regression models were used.

Results: a total of 327 patients in treatment with antithyroid drugs were included. The mean age was $53.7 \pm 18.1$ years and $78.3 \%$ of patients corresponded to women. Methimazole was prescribed in $95.4 \%$ of patients, propylthiouracil in $4.6 \%$. In $76.8 \%$ of patients, comedication was present in particular with antihypertensive agents (38.2\%) and additionally with propranolol $(34.3 \%)$.
Dr. Jorge Enrique Machado-Alba: Profesor Titular de Farmacología y Toxicología, Facultad Ciencias de la Salud. Director Grupo de Investigaciones en Farmacoepidemiología y Farmacovigilancia; Dr. Diego Alejandro Medina-Morales: Grupo de Investigación en Farmacoepidemiología y Farmacovigilancia; Dr. Luis Fernando Valladales-Restrepo: Grupo de Investigación en Farmacoepidemiología y Farmacovigilancia; Dr. Daniel LoaizaBedoya: Grupo de investigación en Farmacoepidemiología y Farmacovigilancia; Dr. Milton Paredes-Mendoza: Grupo de Investigación en Farmacoepidemiología y Farmacovigilancia. Universidad Tecnológica de Pereira-Audifarma S.A. Pereira (Colombia).

Correspondencia. Dr. Jorge Enrique MachadoAlba. Pereira (Colombia).

E-mail: machado@utp.edu.co

Recibido: 8/II/2017 Aceptado: 25/VI/2018 
Conclusions: the prescription tendency of antithyroid drugs in Colombia is similar to that reported in different studies worldwide. The main antithyroid drug is methimazole, with a rate of use higher than that reported in North America and in European studies. The doses of methimazole and propylthiouracil reported in this study are in accordance with the recommendations of the American Association of Clinical Endocrinology. (Acta Med Colomb 2018; 43: 150-155).

Keywords: hyperthyroidism; thyrotoxicosis; antithyroid; methimazole; propylthiouracil; pharmacoepidemiology, (DeCS).

\section{Introducción}

Aunque usados de manera indistinta, los términos hipertiroidismo y tirotoxicosis no son sinónimos (1). El hipertiroidismo hace referencia al exceso de síntesis de hormona tiroidea debido al aumento en la actividad de la glándula tiroidea(2), mientras tirotoxicosis corresponde al síndrome clínico causado por exceso de hormona tiroidea circulante (2-4). A pesar de que la etiología es diversa, se reconoce que cerca de $95 \%$ de los casos son explicados por el bocio tóxico multinodular, el adenoma tóxico y la enfermedad de Graves (2), siendo esta última la causa más común de hipertiroidismo en todos los grupos de edad $(3,5)$, y hasta 10 veces más frecuente en mujeres (2). La prevalencia en la población general es de $0.1-0.5 \%$ (6). El bocio tóxico multinodular típicamente ocurre después de los 50 años, mientras el adenoma, es característico de personas jóvenes (1).

Las manifestaciones clínicas de la tirotoxicosis son muy diversas y dependen de factores como edad del paciente y duración de la enfermedad. Su presentación clínica puede variar desde estados asintomáticos o subclínicos hasta un evento potencialmente fatal como la tormenta tiroidea (1, 7). Los jóvenes suelen presentar signos de estimulación simpática como ansiedad, inquietud y temblor, mientras que los ancianos presentan depresión, fatiga y pérdida de peso, también llamado hipertiroidismo apático $(1,4)$. En cerca de $50 \%$ de los pacientes la debilidad muscular y fatiga fácil constituyen síntomas principales $(1,7)$. La hipertensión sistólica, el incremento de la masa ventricular izquierda, la angina de pecho, y la fibrilación auricular se reconocen como manifestaciones cardiovasculares de la tirotoxicosis (8). El diagnóstico de hipertiroidismo se confirma midiendo TSH y T4 libre $(4,7)$.

Las estrategias de manejo incluyen medicamentos antitiroideos, yodo radioactivo, cirugía de tiroides y tratamiento para el control de síntomas (1). Los medicamentos antitiroideos pueden ser usados como tratamiento primario o como preparación antes de radioterapia o tiroidectomía (9). Son primera elección en pacientes con enfermedad de Graves, aún más en presencia de oftalmopatía (9 -11), así como en gestantes, niños y adolescentes $(1,12,13)$. Las preferencias de tratamiento varían significativamente entre las regiones geográficas. El uso de los medicamentos antitiroideos se reporta en $85.7 \%$ de casos en Europa, alrededor de $70 \%$ en Latinoamérica, Asia y Oceanía, y sólo en 40.5\% de casos de Estados Unidos (13).
En Colombia no se ha establecido la prevalencia de la enfermedad, tampoco se dispone de información de consumo de medicamentos antitiroideos, ni de estudios farmacoepidemiológicos que hayan abordado esta condición, lo cual hace difícil determinar los patrones de prescripción y frecuencia de uso de los diferentes fármacos usados en el tratamiento de la tirotoxicosis (14).

El sistema de salud colombiano ofrece cobertura universal a través de un régimen pago por el contribuyente y su empleador y otro régimen subsidiado por el Estado, que además poseen un plan de beneficios que incluye los medicamentos antitiroideos para todos los pacientes que lo requieran. Ante esta situación, el presente estudio pretendió determinar los patrones de prescripción de los antitiroideos en población colombiana, buscando identificar la tendencia en su uso, dosificación, comedicaciones, potenciales interacciones de riesgo y características socio-demográficas asociadas a la enfermedad y prescripción, con el fin de establecer estrategias de intervención en los casos en que sea necesario, mejorar los conocimientos del manejo farmacológico y adoptar estrategias de seguimiento para la población afectada.

\section{Material y métodos}

Estudio de corte transversal, llevado a cabo entre el 1 de enero y el 30 marzo de 2015 sobre los hábitos de prescripción de medicamentos antitiroideos en una población aproximada de 6.5 millones de personas afiliadas al régimen contributivo del Sistema General de Seguridad Social en Salud de Colombia (SGSSS) a través de cinco entidades promotoras de salud (EPS), correspondiente aproximadamente a $31.0 \%$ de la población afiliada a este régimen en el país y a $13.5 \%$ de la población colombiana.

Se incluyeron los datos de la totalidad de individuos, de cualquier edad y sexo, con dispensación de medicamentos antitiroideos durante al menos tres meses consecutivos, buscando con esto garantizar que los pacientes cumplieran el tratamiento de manera más o menos estable, y sea reflejo de adherencia y tolerabilidad a la medicación; por el contrario se excluyeron las personas que incumplen las citas médicas al final del periodo de observación, considerándose que pueden introducir sesgos en un estudio dirigido a describir patrones de empleo de medicamentos utilizados de manera crónica y continua.

A partir de la información sobre los consumos de medicamentos del operador logístico Audifarma, S.A, empresa 
que dispensa fármacos a la población objetivo, se diseñó una base de datos y se analizaron las siguientes variables:

1. Sociodemográficas (sexo, edad, ciudad).

2. Farmacológicas: nombre del medicamento antitiroideo utilizado, dosis y cantidad dispensada. Se utilizó la DDD como unidad de medida de la utilización de los siguientes fármacos, según las recomendaciones de la Organización Mundial de la Salud (OMS) y expresada como DHD (dosis diaria definida por cada 1.000 habitantes por día). Los costes unitarios totales y el costo por 1000 habitantes/día $(\mathrm{CHD}=($ costo/365 x No. habitantes $) \times(1000)$ fueron utilizados para estimar el impacto económico del uso de fármacos antitiroideos, utilizando el precio mínimo o el máximo de mercado y el precio de referencia de la compañía de distribución para diferentes compañías de seguros (EPS). Además, se estimaron los costos mensuales y anuales de estas prescripciones.

3. Se aceptó la comedicación como un indicador sustituto de la enfermedad crónica, considerando las siguientes circunstancias:

- Se identificaron los diagnósticos principales asociados a cada prescripción y se utilizó la comedicación como un indicador sustituto de enfermedad crónica asociada, así: antihipertensivos y diuréticos/hipertensión arterial, antidiabéticos/diabetes mellitus, antiagregantes plaquetarios/ riesgo cardiovascular nitratos/enfermedad coronaria, analgésicos/dolor, amiodarona o propafenona/arritmias, digitálicos/ insuficiencia cardiaca o fibrilación auricular, antiulcerosos/enfermedad ácido peptica, hipolipemiantes/ dislipidemia, antidepresivos/depresión, antidemenciales/demencia y enfermedad de Alzheimer; broncodilatadores/enfermedad pulmonar obstructiva crónica; ansiolíticos e hipnóticos/trastornos de ansiedad o sueño, estabilizantes del afecto/trastorno afectivo bipolar y p) antipsicóticos/psicosis-esquizofrenia.

- Se revisaron las combinaciones de medicamentos que pudieran ocasionar interacciones potencialmente riesgosas con el propósito de hacer recomendaciones al ente responsable de la atención del paciente.

Para el análisis de los datos, se utilizó el paquete estadístico SPSS, versión 23.0 (IBM, EE. UU.) para Windows. Se emplearon las pruebas t de Student o ANOVA para la comparación de variables cuantitativas y la de $X^{2}$ para las categóricas. Se aplicaron modelos de regresión logística binaria usando como variables dependientes la terapia única con antitiroideos vs terapia combinada y la comedicación (sí/no), y como covariables las que se asociaron significativamente con las variables dependientes en los análisis bivariados. Se determinó como nivel de significancia estadística una $\mathrm{p}<0.05$.

El trabajo contó con el aval del Comité de Bioética de la Universidad Tecnológica de Pereira y se clasificó en la categoría de investigación sin riesgo respetando los principios de confidencialidad de la Declaración de Helsinki.

\section{Resultados}

A partir de la base de datos se halló un total de 327 pacientes en tratamiento con medicamentos antitiroideos por un periodo mínimo de tres meses. La edad media fue de $53.7 \pm 18.1$ años (rango: $12-95$ años) y $78.3 \%(n=256)$ de casos correspondió a mujeres. La relación mujer: hombre en la población de estudio fue de 3.6:1. En la Figura 1 se muestra la distribución por edad y sexo y en la Tabla 1 se resumen las características sociodemográficas, clínicas y farmacológicas de los pacientes.

El metimazol se prescribió en $95.4 \%$ de los casos $(n=312)$, mientras el propiltiouracilo en $4.6 \%(n=15)$. Ninguno de los pacientes, tenía prescripción con estos medicamentos en forma concomitante. El patrón de prescripción de los medicamentos antitiroideos se resume en la Tabla 2.

\section{Comedicación}

El $76.8 \%$ de pacientes $(n=251)$ recibían concomitantemente uno o varios medicamentos para otras enfermedades, que pueden presentar posibles interacciones con algunos de los medicamentos antitiroideos. De los pacientes que recibían metimazol, el $77.6 \%(\mathrm{n}=242)$ tenían comedicaciones, mientras que en aquellos con propiltiouracilo fue $60.0 \%$ $(n=9)$ (Tabla 1). El promedio de fármacos por paciente fue $2.4 \pm 2.3$, (rango 0 - 10 fármacos).

\section{Comparación entre ciudades}

Se compararon las variables demográficas y algunos indicadores de prescripción entre las 37 ciudades o municipios colombianos incluidos en este estudio, no obstante, por razones relacionadas con el bajo número de pacientes en algunas de ellas, en la Tabla 1 sólo se incluyeron las siete principales, donde reside el $83.5 \%$ de los pacientes y se hizo el mismo análisis para todo el país.

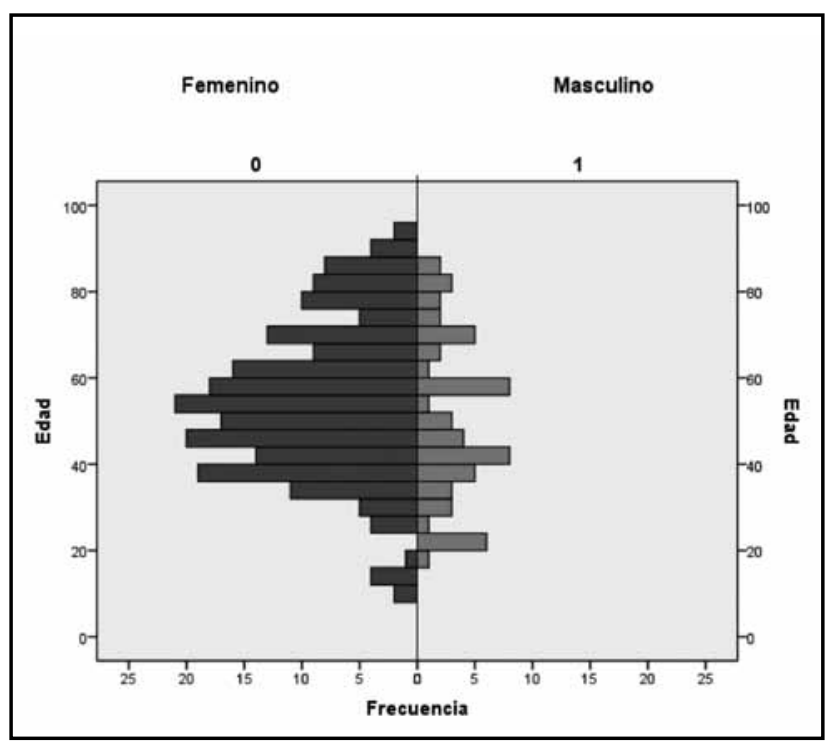

Figura 1. Distribución por edad y sexo de 327 pacientes con hipertiroidismo en tratamiento con metimazol o propiltiouracilo y afiliados al Sistema de Salud de Colombia, 2015. 
TRABAJOS ORIGINALES • Patrones de prescripción de antitiroideos

Tabla 1. Características de los 327 pacientes tratados con antitiroideos en población de Colombia, 2015.

\begin{tabular}{|c|c|c|c|c|c|c|}
\hline \multirow{3}{*}{ Variable } & \multicolumn{2}{|c|}{ Población general } & \multicolumn{2}{|c|}{ Metimazol } & \multicolumn{2}{|c|}{ Propiltiouracilo } \\
\hline & Frecuencia & Porcentaje & Frecuencia & Porcentaje & Frecuencia & Porcentaje \\
\hline & $(\mathrm{n}=327)$ & $(\%)$ & $(n=312)$ & $(\%)$ & $(n=15)$ & $(\%)$ \\
\hline Edad: media \pm desviación estándar & $53.7 \pm 18.1$ & & $53.7 \pm 18.0$ & & $55.7 \pm 19.5$ & \\
\hline Sexo: Mujeres/hombres & $256 / 71$ & $78.3 / 21.7$ & $243 / 69$ & $77.9 / 22.1$ & $13 / 2$ & $86.7 / 13.3$ \\
\hline \multicolumn{7}{|l|}{ Procedencia } \\
\hline Bogotá & & & 147 & 94.8 & 8 & 5.2 \\
\hline Cali & & & 50 & 100.0 & 0 & 0.0 \\
\hline Barranquilla & & & 32 & 91.4 & 3 & 8.6 \\
\hline Cartagena & & & 12 & 100.0 & 0 & 0.0 \\
\hline Pereira & & & 8 & 88.9 & 1 & 11.1 \\
\hline Santa Marta & & & 7 & 100.0 & 0 & 0.0 \\
\hline Tuluá & & & 5 & 100.0 & 0 & 0.0 \\
\hline \multicolumn{7}{|l|}{ Diagnóstico principal } \\
\hline Tirotoxicosis & 35 & 33.7 & 33 & 33.3 & 2 & 15.4 \\
\hline Trastorno de la glándula tiroides & 19 & 18.3 & 19 & 19.2 & 0 & 0.0 \\
\hline Compromiso oftálmico y otros & 11 & 10.6 & 10 & 10.1 & 1 & 7.7 \\
\hline Otros (no relacionados a tiroides) & 39 & 37.5 & 37 & 37.4 & 10 & 76.9 \\
\hline \multicolumn{7}{|l|}{ Comedicación } \\
\hline Antihipertensivos & 125 & 38.2 & 118 & 37.8 & 7.0 & 46.7 \\
\hline Propranolol & 112 & 34.3 & 108 & 34.6 & 4.0 & 26.7 \\
\hline Otros medicamentos cardiovasculares & 109 & 33.3 & 104 & 33.3 & 5.0 & 33.3 \\
\hline Analgésicos & 94 & 28.7 & 90 & 28.8 & 4.0 & 26.7 \\
\hline Antiulcerosos & 69 & 21.1 & 66 & 21.2 & 3.0 & 20.0 \\
\hline Antidepresivos & 33 & 10.1 & 29 & 9.3 & 4.0 & 26.7 \\
\hline Antidiabéticos & 31 & 9.5 & 29 & 9.3 & 2.0 & 13.3 \\
\hline Tratamiento EPOC*/asma & 10 & 3.1 & 10 & 3.2 & 0.0 & 0.0 \\
\hline Ansiolíticos/hipnóticos & 10 & 3.1 & 9 & 2.9 & 1.0 & 6.7 \\
\hline Promedio de medicamentos recibidos & $2.7 \pm 2.5$ & & $2,6 \pm 2,5$ & & $2.7 \pm 2.6$ & \\
\hline
\end{tabular}

Tabla 2. Patrones de uso de antitiroideos en 327 pacientes afiliados al Sistema de Salud de Colombia, 2015.

\begin{tabular}{|c|c|c|c|c|c|c|c|}
\hline \multirow{2}{*}{ Medicamento } & \multicolumn{2}{|c|}{ Frecuencia } & \multicolumn{2}{|c|}{ Dosis prescrita (mg/día) } & \multirow{2}{*}{$\frac{\text { Relación sexo }}{\text { (Mujer:Hombre) }}$} & \multirow{2}{*}{$\frac{\text { Edad }}{(\text { años } \pm D E)}$} & \multirow{2}{*}{$\begin{array}{c}\text { Relación } \\
\text { Dosis media } \\
\text { prescrita/DDD }\end{array}$} \\
\hline & (n) & $(\%)$ & Media & Moda & & & \\
\hline Metimazol & 312 & 95.4 & $15.9 \pm 11.2$ & 10 & $3.5: 1$ & $53.7 \pm 18.0$ & 1.6 \\
\hline Propiltiouracilo & 15 & 4.6 & $222.8 \pm 182.9$ & 100 & $6.5: 1$ & $55.7 \pm 19.5$ & 1.0 \\
\hline
\end{tabular}

\section{Análisis multivariado}

El análisis multivariado mostró que las variables tener entre 21 - 44 años (OR:0.450; IC95\%:0.252-0.804; $p=0.007$ ), y residir en la ciudad de Barranquilla (OR:0.413; IC95\%:0.194-0,882 $p=0.022$ ) se asociaron estadísticamente con una menor probabilidad de recibir comedicación, mien- tras que la edad mayor de 65 años (OR:2.55; IC95\%:1,077$6.054 ; p=0.033$ ) se asoció a un aumento en el riesgo de esto.

\section{Discusión}

El presente estudio permitió documentar los patrones de prescripción de medicamentos antitiroideos, las come- 
dicaciones más frecuentes y otras variables asociadas, en un grupo de pacientes afiliados al SGSSS de Colombia. Estos hallazgos son de utilidad para el personal asistencial y científico, ya que permite de manera indirecta conocer la epidemiología de la enfermedad y establece un antecedente para los sistemas de farmacovigilancia.

La relación por sexo mostró un predomino femenino siendo similar a lo encontrado en otros estudios $(5,6)$. En esta investigación se encontró una edad media de 53.7 años, lo que es comparable con lo reportado por Azevedo en Brasil, quien halló un predominio femenino (86.9\%) y una edad media de 54.0 años (5).

En las últimas décadas han existido diferencias notables a nivel internacional respecto al modo de manejo del hipertiroidismo debido a enfermedad de Graves (5). Las estrategias de manejo incluyen medicamentos antitiroideos, yodo radioactivo, cirugía tiroidea y tratamiento farmacológico para el control de síntomas (1); no obstante, los medicamentos antitiroideos constituyen la primera línea de manejo (11). En un estudio sobre patrones de manejo de la enfermedad de Graves (11) se observó que el tratamiento con antitiroideos fue el método preferido en $54.9 \%$ de casos (10). Las preferencias de manejo comparadas por región geográfica son significativamente diferentes, con menor uso de estos fármacos en Estados Unidos (40.5\%) que en Europa (85.7\%), Latinoamérica (73.8\%), y Asia/Oceanía (70.6\%) (10). Existe consenso que durante el primer trimestre de la gestación y la lactancia el medicamento de elección es propiltiouracilo, el cual puede reemplazarse por metimazol en el segundo y tercer trimestre del embarazo $(5,15)$, que además es el medicamento de elección para el tratamiento del hipertiroidismo en niños y adolescentes (16).

La frecuencia de prescripción de antitiroideos en nuestro país es similar a lo reportado en diferentes estudios llevados a cabo en Europa y Norteamérica $(10,12)$. El principal fármaco utilizado en Colombia es el metimazol, prescrito al $95.4 \%$ de los pacientes lo que representa una tasa de uso mayor a la reportada en Norteamérica $(83.5 \%)$ y en estudios europeos (79.3\%), regiones en las que el carbimazol se formula en 13.8 - $17.9 \%$ de pacientes; sin embargo, dicho medicamento no se encuentra disponible en nuestro país (17). La frecuencia de uso del propiltiouracilo a nivel global, es inferior a 5\% (17), lo cual fue encontrado también en nuestro estudio.

Las dosis del metimazol y de propiltiouracilo reportadas en este estudio son similares a lo previamente descrito en Europa (13) y que se ajusta a las recomendaciones de la Asociación Americana de Endocrinología Clínica (18). Tanto el metimazol como el propiltiouracilo son medicamentos que se asocian a una alta tasa de reacciones adversas medicamentosas (RAM) (19), entre las que destacan por su frecuencia, rash, urticaria, fiebre y artralgias, mientras la agranulocitosis es la más temida. Adicionalmente el propiltiouracilo se ha asociado a falla hepática severa (20), lo cual ha relegado su uso a casos muy específicos (7). En un estudio con 391 pacientes se compararon dosis de metimazol a razón de 15 $\mathrm{mg} / \mathrm{d}$ á, $30 \mathrm{mg}$ /día y propiltiouracilo de $300 \mathrm{mg} /$ día, encontrándose menor frecuencia de hepatotoxicidad y reacciones cutáneas en el primer grupo (21). Sin embargo, en el presente trabajo no se pudo definir las RAM que presentaron los pacientes en tratamiento con antitiroideos.

El propranolol fue la comedicación más común y se presume fue utilizado para el control de los síntomas asociados al hipertiroidismo. El uso del propranolol es reconocido en el tratamiento de la enfermedad, y aunque no modifica el curso de la misma, permite el control de los signos y síntomas hasta que se logre el estado eutiroideo (6).

Entre las posibles interacciones medicamentosas de riesgo, los antitiroideos pueden disminuir los efectos de los anticoagulantes del grupo de antagonistas de la vitamina K1. En el presente estudio sólo se identificaron dos pacientes formulados simultáneamente con metimazol y warfarina. La presencia de estos casos obliga al análisis individual para determinar si la asociación es apropiada o no, buscando reducir el riesgo de daños y garantizar una anticoagulación efectiva de acuerdo a las recomendaciones específicas de la condición por tratar (22).

En el hipertiroidismo la elevación de la tensión arterial es una condición usual, siendo de predominio sistólico $(8,23)$. En este estudio se encontró que la hipertensión arterial fue la comorbilidad más frecuente (17.4\%) y un bajo porcentaje de pacientes presentaron diabetes mellitus. Estos datos son congruentes con estudios regionales; en Brasil la hipertensión fue la comorbilidad más común, encontrándose en $29.5 \%$ de los pacientes con diagnóstico de enfermedad de Graves (5). Se estima que la prevalencia de la hipertensión arterial en pacientes con tirotoxicosis varía entre 20 y $30 \%$, sin embargo, los estudios que confirmen estos datos son limitados, debido a la alta prevalencia de la primera en la población general $(24,25)$. Por su parte, se reconoce que la diabetes mellitus es un factor de riesgo importante para el agravamiento de estas comorbilidades (5); en un estudio europeo se encontró que este trastorno metabólico tuvo una prevalencia de $6,4 \%$ en pacientes con enfermedad de Graves (26); cifras superiores a las halladas en el presente estudio. Dado que un importante número de pacientes padece simultáneamente (hipertiroidismo, hipertensión arterial y/o diabetes mellitus), suponen un grupo de alto riesgo cardiovascular, sobre el cual deben realizarse intervenciones de prevención que disminuyan la probabilidad de eventos coronarios, cerebrovasculares, falla cardiaca y otras complicaciones (27).

Este estudio permite inferir la epidemiología de la enfermedad y ofrece el panorama nacional de uso de medicamentos para el tratamiento de los desórdenes hipertiroideos, ya que se realizó en una amplia base de datos poblacional. No obstante, se reconocen algunas limitaciones en la interpretación de ciertos resultados, puesto que no se tuvo acceso a las historias clínicas, lo cual impidió la identificación de las causas de hipertiroidismo las cuales además varían en frecuencia entre los diferentes grupos etarios $(4,13)$; tampoco 
se tuvo acceso a los resultados de los exámenes paraclínicos que indiquen el nivel de control del hipertiroidismo, así como no se pudo obtener información de la presentación de reacciones adversas. Sin embargo, éstas pueden solucionarse con el desarrollo de estudios posteriores que busquen el diagnóstico etiológico de la condición.

Se puede concluir que los pacientes con hipertiroidismo afiliados al SGSSS que reciben tratamiento farmacológico son manejados predominantemente con metimazol a dosis adecuadas, además, reciben propranolol como terapia de apoyo y con frecuencia sufren hipertensión arterial, diabetes mellitus, depresión. Ante la carencia de estudios previos en Colombia sobre prevalencia de la enfermedad y patrones de prescripción en medicamentos antitiroideos (14), éste se constituye en un antecedente de importancia para los sistemas de farmacovigilancia nacionales y servirá de marco para futuras investigaciones que permitan una caracterización adicional de la patología.

\section{Agradecimientos}

A Soffy López, Andrea Orozco y Viviana Orozco por su apoyo en la obtención de la base de datos

\section{Conflicto de intereses}

Los autores expresan que no existe ningún conflicto de intereses.

\section{Fuentes de financiación}

El presente trabajo no recibió fuentes de financiación.

\section{Referencias}

1. Devereaux D, Tewelde SZ. Hyperthyroidism and Thyrotoxicosis. Emerg Med Clin North Am. 2014;32(2):277-92.

2. Truran P, Aspinall S. Thyrotoxicosis and thyroiditis. Surgery (Oxf). 2014;32(10):537-42.

3. Sharma M, Aronow WS, Patel L, Gandhi K, Desai H. Hyperthyroidism. Med Sci Monit. 2011;17(4):85-91.

4. Seigel SC, Hodak SP. Thyrotoxicosis. Med Clin North Am. 2012;96(2):175-201.

5. Ross DS. Radioiodine therapy for hyperthyroidism. $N$ Engl J Med. 2011;364(6):542-50.

6. Fadel A, Gutiérrez S, Novelli JL, Orlandi AM, Parma R, Croome S, et al. Tratamiento del hipertiroidismo por Enfermedad de Graves en pacientes adultos no embarazadas. Rev Argent Endocrinol Metab. 2013;50(2):107-26.

7. Franklyn JA, Boelaert K. Thyrotoxicosis. Lancet. 2012;379(9821):1155-66.
8. Grais IM, Sowers JR. Thyroid and the heart. Am J Med. 2014;127(8):691-8.

9. Menconi F, Marcocci C, Marinò M. Diagnosis and classification of Graves' disease. Autoimmun Rev. 2014;13(4-5):398-402.

10. Burch HB, Burman KD, Cooper DS. A 2011 survey of clinical practice patterns in the management of Graves' disease. J Clin Endocrinol Metab. 2012;97(12):4549-58.

11. Cooper DS. Antithyroid drugs. N Engl J Med. 2005;352(9):905-17.

12. Bartalena L, Burch HB, Burman KD, Kahaly GJ. A 2013 European survey of clinical practice patterns in the management of Graves' disease. Clin Endocrinol (Oxf). 2016;84(1):115-20.

13. García Botina HD, Córdoba Ramírez N, Builes Barrera CA. Diagnóstico y tratamiento de los trastornos tiroideos durante el embarazo: un enfoque para el cuidado integral del binomio madre-hijo. Iatreia. 2013;26(2):172-84.

14. Asociación Colombiana de Endocrinología. Consenso colombiano para el diagnóstico y manejo de las enfermedades tiroideas. Acta Med Colomb. 1999;24:15974.

15. Azevedo FV, Blotta FG, Goirgetta JM, Noe R, Vaisman M. Emerging comorbidities in Graves' disease patients treated with radioiodine with more than 10 years of follow-up. Arq Bras Endocrinol Metabol. 2013;57(1):51-6.

16. Calagua Quispe M, Núñez Almache O, Falen Boggio J, Del Águila Villar C, Lu de Lama R, Rojas Gabulli MI, et al. Respuesta al tratamiento de la Enfermedad de Graves-Basedow en pacientes pediátricos. An Fac Med Lima. 2014;75(2):13136.

17. Colombia Ministerio de Salud. Resolución 5926 de 2014. Diario Oficial Disponible en: https://http://www.minsalud.gov.co/salud/POS/mi-plan/Paginas/ Mi-Plan.aspx.Consultado: Octubre 20, 2016

18. Bahn Chair RS, Burch HB, Cooper DS, Garber JR, Greenlee MC, Klein I, et al. Hyperthyroidism and other causes of thyrotoxicosis: management guidelines of the American Thyroid Association and American Association of Clinical Endocrinologists. Thyroid. 2011;21(6):593-646.

19. Labadzhyan A, Brent GA, Hershman JM, Leung AM. Thyrotoxicosis of pregnancy. J Clin Transl Endocrinol. 2014;1(4):140-4.

20.Gargallo Fernández M. Hyperthyroidism and pregnancy. Endocrinol Nutr. 2013;60(9):535-43.

21. Otsuka F, Noh JY, Chino T, Shimizu T, Mukasa K, Ito K, et al. Hepatotoxicity and cutaneous reactions after antithyroid drug administration. Clin Endocrinol (Oxf). 2012;77(2):310-15.

22. Yurgaky J, Rodríguez F. Warfarina: uso contemporáneo. Rev Fac Med 2009;17(1):107-15.

23. Prisant LM, Gujral JS, Mulloy AL. Hyperthyroidism: a secondary cause of isolated systolic hypertension. J Clin Hypertens (Greenwich). 2006;8(8):596-9.

24. Cooper DS, Rivkees SA. Putting propylthiouracil in perspective. J Clin

Endocrinol Metab. 2009;94(6): 1881 .

25. Mazza A, Beltramello G, Armigliato M, Montemurro D, Zorzan S, Zuin M, et al. Arterial hypertension and thyroid disorders: What is important to know in clinical practice?. Ann Endocrinol (Paris). 2011;72(4):296-303.

26. Le Moli R, Muscia V, Tumminia A, Frittitta L, Buscema M, Palermo F, et al. Type 2 diabetic patients with Graves' disease have more frequent and severe Graves' orbitopathy. Nutr Metab Cardiovasc Dis. 2015;25(5):452-7.

27. Mantilla D, Echin ML, Perel C. Hipertiroidismo y sistema cardiovascular: Bases fisiopatológicas y su manifestación clínica. Insuf Card. 2010;5(4):157-77. 